\title{
MULTI-Hop DisTRIBUTED ENERGY EFFICIENT Hierarchical Clustering Scheme For Heterogeneous Wireless SENSOR NeTWORKS
}

\author{
Sheenam \\ M. Tech Research Scholar \\ Department of Electronics and communication Engineering, Punjabi University, Patiala, \\ India
}

\begin{abstract}
Wireless sensor network (WSNs) are network of Sensor Nodes (SNs) with inherent sensing, processing and communicating abilities. One of current concerns in wireless sensor networks is developing a stable clustered heterogeneous protocol prolonging the network lifetime with minimum consumption of battery power. In the recent times, many routing protocols have been proposed increasing the network lifetime, stability in short proposing a reliable and robust routing protocol. In this paper we study the impact of hierarchical clustered network with sensor nodes of two-level heterogeneity. The main approach in this research is to develop an enhanced multi-hop DEEC routing protocol unlike DEEC. Simulation results show the proposed protocol is better than DEEC in terms of FDN (First Dead Node), energy consumption and Packet transmission.
\end{abstract}

\section{KEYWORDS}

Wireless Sensor Networks, FDN, DEEC, Heterogeneity, Intermediate Nodes, Network Lifetime.

\section{INTRODUCTION}

Wireless sensor networks are composed of thousands or more sensor nodes which are distributed manually or randomly in physical sensor environment to sense the characteristics of environment. The main aim behind such deployment is to fulfill some particular aim of particular application such as acoustics, temperature, humidity, light, seismic etc [1]. Network collect the environment data and forward to processing nodes for further processing. These nodes in high density are deployed in buildings, homes, highways, cities [2]. The range of applications is from warning natural disasters, protecting homeland, conducting surveillance, wildlife monitoring, environment monitoring etc. These sensor nodes are considered as energy scarce system resources as they have short battery life [3]. The cost is more in transmission of data than processing of data so if there can be a scheme where this transmission distance is decreased with some sort of changes in deployment, it would be a boon for the system.

In Direct transmission (DT), the data from sensor nodes (SNs) is transmitted directly to BS (Base Station). In such transmission if the Bs is located far away from SNs, the sensor nodes away would require more energy than nearby sensor nodes in DT. Similarly in MTE (Minimum Transmission Energy), sensor nodes are sending data to neighboring sensor nodes and finally 
reach BS, here the nodes near to BS are more participating in data transmission than faraway nodes, so they are draining their energy faster and die faster as compared to other nodes [4]. Even there is no resource for recharging of sensor nodes after particular time period if they are not been installed with energy recharging resources. So maximizing the network lifetime with energy efficiency is current challenge that we are facing in this field.

Clustering [2] is a technique used for reducing energy usage by assigning a Cluster Head $(\mathrm{CH})$ for aggregating data and forwarding this aggregated data to BS. $\mathrm{CH}$ here is acting as intermediate node between sensor nodes and BS. Flat network is network of sensor nodes with no $\mathrm{CH}$ and no cluster formation and each sensor node is sending its data to other node or to BS but clustered network is network of clusters with one $\mathrm{CH}$ of each cluster for data transmission as shown in figure 1[5]. In Clustered network, the whole network is composed of group of sensor nodes called as cluster with one head of family (cluster) who is responsible for data communication with other BS.

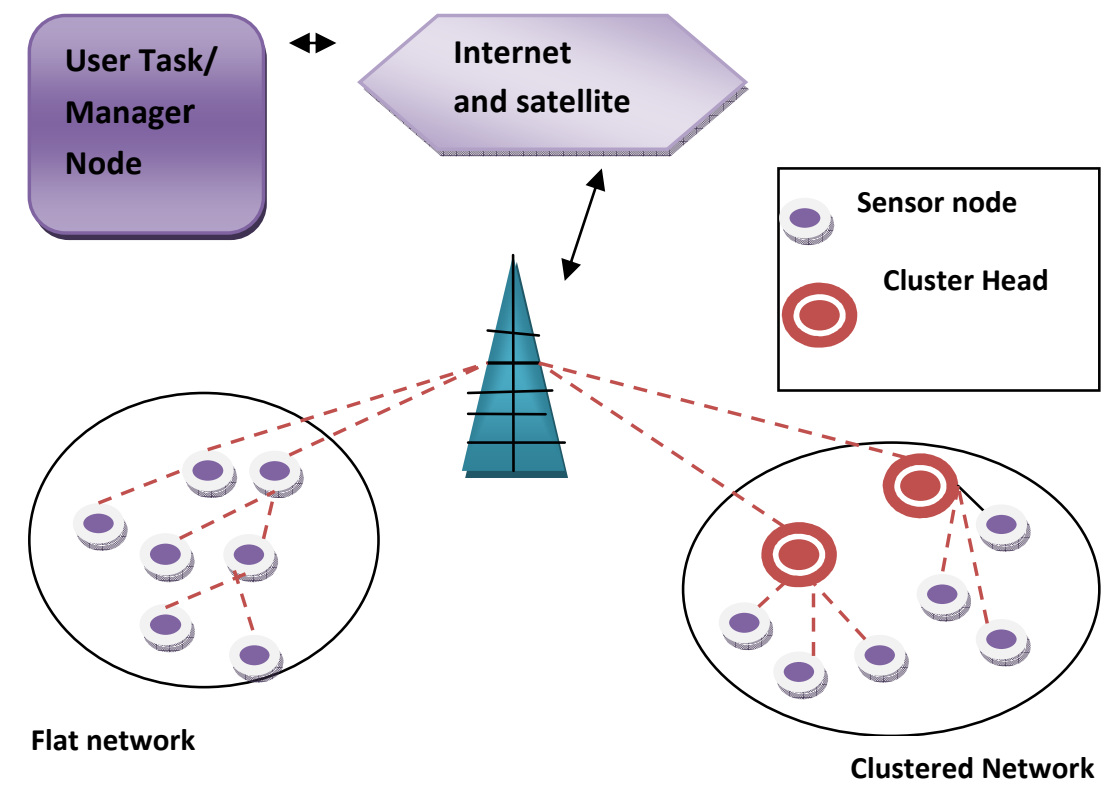

Figure 1. Network operations of WSNs

Even after clustering still wireless sensor networks are suffering from un-efficient energy management. So in this research we are proposing a large scale multi-hop network system infrastructure comprising of sensor nodes having low battery power and processing, computing capabilities. Intermediate rechargeable nodes are deployed in network field for enhancing the lifetime of networks. Intermediate nodes so placed are decreasing the average distance of data transmission form sensor nodes to BS.

\section{RELATED WORK}

LEACH [4] was first clustered homogeneous network scheme which uses direct transmission of data from $\mathrm{CH}$ selected randomly to BS leads to draining of energy of sensor nodes quickly. Prior selecting is not done as it may lead to some unfavorable nodes becoming $\mathrm{CHs}$ that would die 
quickly. LEACH outperforms DT and MTE in terms of network stability. PEGASIS [6] was a chain based routing scheme provides improvement over LEACH scheme. Each node communicates with nearby node and take turns of transmission until reaches BS. PEGASIS was twice better than LEACH in terms of network lifetime. HEED [7] was proposed as an enhancement over homogeneous protocols like LEACH, PEGASIS, TEEN etc where $\mathrm{CH}$ was selected based on node residual energy and also on basis of node proximity to nearby node i.e. node's degree. HEED outperforms other routing protocols. All the above protocols described are homogeneous considering each node of similar architecture, computing capabilities and initial energy. But then comes the SEP [8] where discrimination in nodes in terms of energy is considered i.e. higher energy nodes are advance nodes and low energy nodes are normal nodes. $\mathrm{CH}$ is chosen based on initial energy of nodes. SEP outperforms the other protocols like LEACH in terms of network stability. DEEC [9] is also a heterogeneous clustered routing protocol where $\mathrm{CHs}$ are chosen based on node's residual energy and DEEC outperforms SEP in terms of stability. DDEEC [10] is another advancement of DEEC which permits to balance the $\mathrm{CH}$ selection over the whole network based on residual energy. H-SEP [11] (Heterogeneous SEP) is an advancement over SEP introducing two level heterogeneity in terms of node energy. H-SEP outperforms SEP in terms of lifetime. SEP-E [12] (Enhanced SEP) was proposed as an advancement of SEP where three-level heterogeneity is been considered. Three types of nodes with different energy levels is been taken. SEP-E outperforms LEACH and SEP in terms of FDN and throughput. HSEP [13] is a heterogeneity aware hierarchical stable election protocol which tends to increase the lifetime of WSNs by choosing two types of CHs i.e. primary and secondary CHs. Primary $\mathrm{CH}$ are taken from secondary ones. HSEP outperforms SEP, LEACH, DEEC by $75 \%, 61 \%, 52 \%$ resp. M-GEAR [14] for WSNs introduced gateway node at center of network and BS out of field. Simulation results showed M-GEAR outperforms LEACH in terms of network lifetime, throughput etc. ZSEP [15] uses both the direct transmission and clustering. ZSEP divide the sensor network in three zones depending on distance from BS. ZSEP outperforms SEP in terms of stability of network. MDEEC [16] modifies the existing DEEC allowing more number of data to be transferred to BS in particular time interval. Simulation results showed MDEEC has incremented message transmission when compared to existing LEACH and DEEC protocol. SEARCH [17] was proposed to increase the number of half alive nodes and stability. SEARCH (Stochastic election of appropriate range cluster heads) updates the $\mathrm{G}$ value quickly making the availability of nodes all the time for becoming $\mathrm{CHs}$.

\section{PROPOSED DEEC (IDEEC PROTOCOL)}

The basic assumptions of new proposed IDEEC model are as:

1. The BS is kept at $35 \mathrm{~m}$ distance away from $100 \mathrm{~m} * 100 \mathrm{~m}$ sensor network field.

2.16 intermediate nodes are placed inside the network field in a proper manner.

3. Intermediate nodes so placed are rechargeable.

4. Each sensor node is equipped with GPS to make them location aware.

5. BS also is aware of its position in field and position of sensor nodes.

6. Distance to be covered for transmission is reduced with aid of these intermediate nodes.

7. Each Sensor node is equipped with one distinct location ID. 


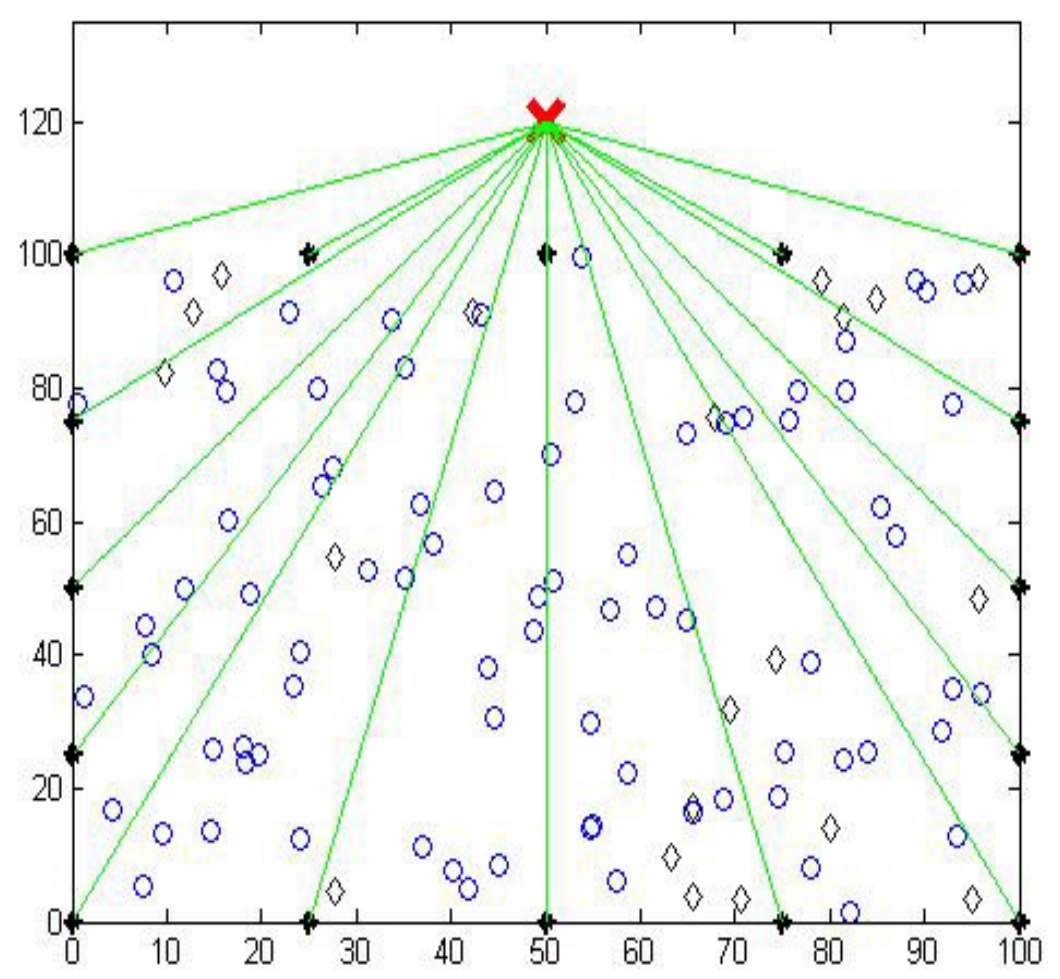

Figure 2. Simulation Network Model of IDEEC
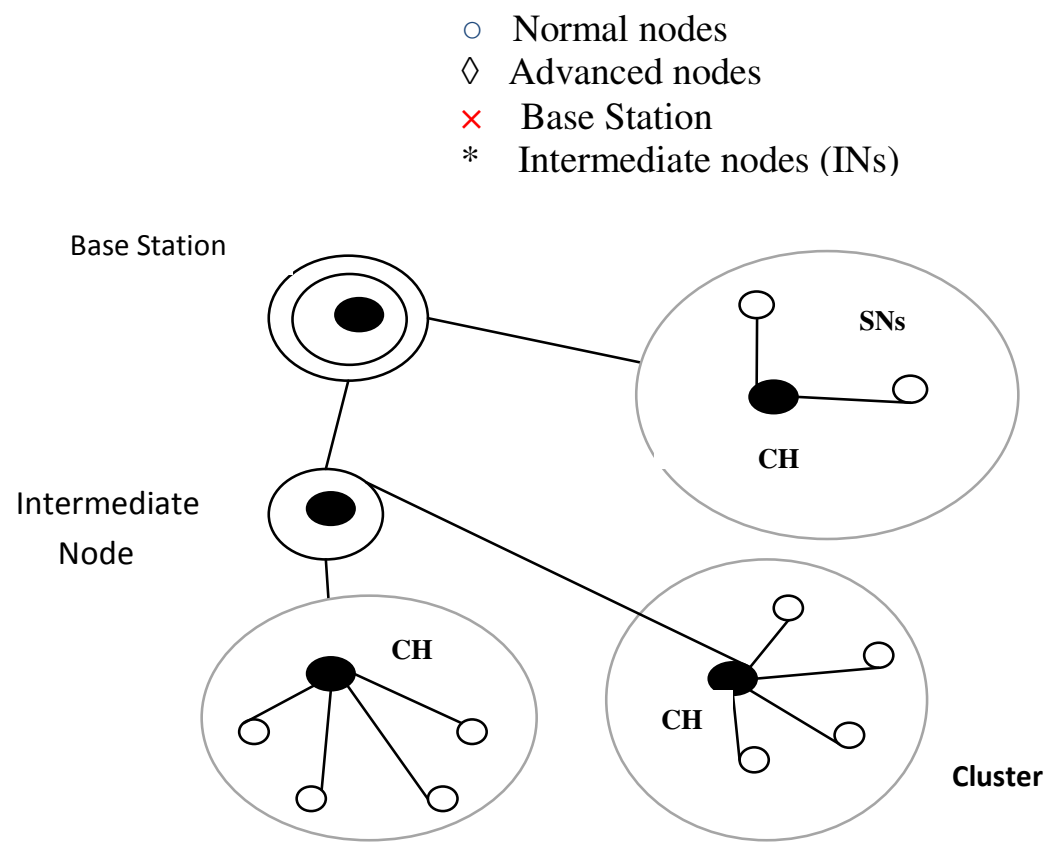

Figure 3. Schematic model of IDEEC 
Here in this research a large scale multi-hop network system we consider four types of sensor nodes i.e. Normal Nodes, Advance Nodes and Intermediate Nodes (INs) and final Base Station (BS) Node shown in figure 3.

Normal nodes and advance nodes are having limited battery power and are non-rechargeable whereas the Intermediate nodes are rechargeable and have unlimited battery power. More over the BS so placed is stationary as well as rechargeable. Sensor nodes form clusters and send data to their $\mathrm{CH}$. $\mathrm{CH}$ discovers the nearby intermediate node and forward data to BS. The number of intermediate nodes placed at four boundaries of network field so the data out of field has to go through these intermediate nodes and then to BS. One intermediate node is receiving data from one $\mathrm{CH}$ data time and incase of failure of one intermediate node, data can be forwarded from another intermediate node. The main function of intermediate node (INs) is to collect data from $\mathrm{CHs}$ and forward that aggregated data to BS.

Here we used radio propagation model as data is transmitted through Radio, infra-red or optical media in wireless sensor networks. This model was proposed in LEACH and SEP [4, 7] shown in figure 4. The energy expended by radio in achieving minimum SNR level in transmitting 4000 bits long packet bits from transmitter to receiver is given as

$$
E_{T X}(l, d)=\left\{\begin{array}{c}
L \cdot E_{\text {elec }}+L \in_{f s} \cdot d^{2}, \text { ifd } \leq d_{o} \\
L . E_{\text {elec }}+L \in_{m p} \cdot d^{4}, \text { ifd }>d_{o}
\end{array}\right.
$$

Where, $E_{\text {elec }}$ is the energy dissipated per bit to run the transmitter or the receiver circuit, $\epsilon_{f s}$ and $\epsilon_{m p}$ depends on the transmitter amplifier model, and $\mathrm{d}$ is the distance between the sender and the receiver.

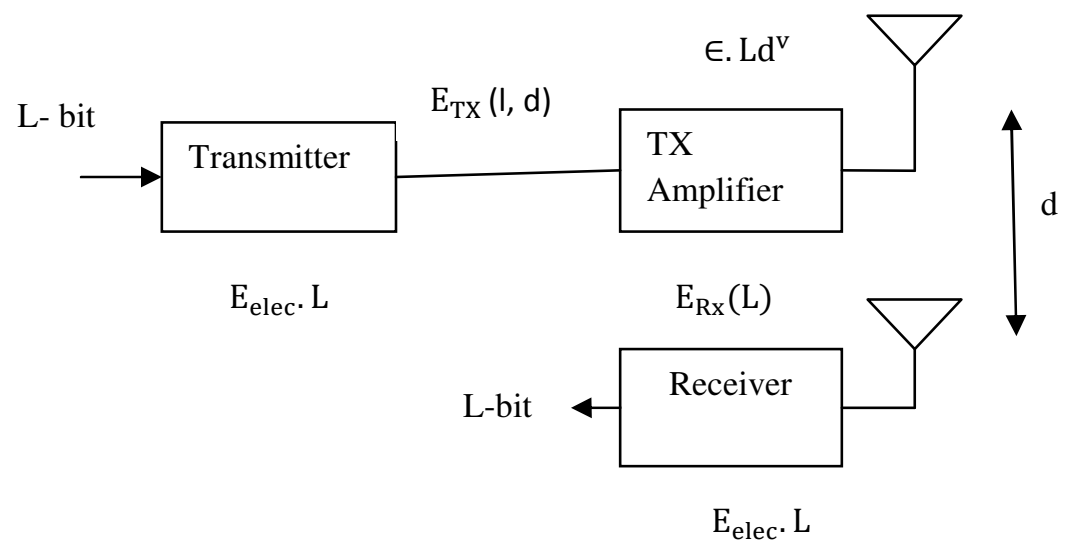

Figure 4. Radio dissipation model

Model considers transmitter, amplifier and receiver. L-bit data is been transmitted from sender to receiver. $d_{o}$ is threshold distance between two communicating ends.

At $d=d_{o}$

$$
d_{o}=\sqrt{\frac{\epsilon_{f s}}{\epsilon_{m p}}}
$$




\section{CH SELECTION ALGORITHM OF IDEEC}

In this research multi-hop DEEC using INs is proposed. The cluster head of each cluster is selected on basis of ratio of residual energy to average energy [8]. Different probability of $\mathrm{CH}$ selection is their i.e. for normal nodes and advance nodes. Selection of INs is done based on distance from selected $\mathrm{CH}$. Even the sensor nodes can send data directly to BS if their distance from BS is less than $\mathrm{CH}$ or IN. Also $\mathrm{CH}$ can send data directly to BS if their distance from BS is less than from IN. Average energy is ideal energy that each node shown to make system alive for longer time. Average energy is given as

$$
\mathrm{E}_{\mathrm{a}}=\frac{1}{\mathrm{~N}} \sum_{\mathrm{i}=1}^{\mathrm{N}} \mathrm{E}_{\mathrm{i}}
$$

Where $E_{i}$ is residual energy of each sensor node. $\mathrm{N}$ is number of sensor nodes deployed. The weighted election probability of normal nodes $\mathrm{p}_{\mathrm{nrm}}$ and advance nodes $\mathrm{p}_{\mathrm{adv}}$ is given as

$$
\begin{aligned}
& p_{n r m}=\frac{p_{o p t}}{1+m 1 \times \alpha 1} \times \frac{E_{i}}{E_{a}} \\
& p_{a d v}=\frac{p_{o p t}}{1+\mathrm{m} 1 \times a 1} \times(1+a 1) \times \frac{E_{i}}{E_{a}}
\end{aligned}
$$

where $\mathrm{m} 1$ is fraction of advance nodes having $\alpha 1 \%$ extra energy in comparison to $\mathrm{m}$ fraction of advance nodes with $\alpha \%$ additional energy in DEEC [8]. $\mathrm{p}_{\text {opt }}$ is the optimal probability assumed in priori.

After selecting the $\mathrm{CHs}$ from each processing round, a random number is generated based on which the $\mathrm{CH}$ is chosen from those selected $\mathrm{CHs}$. After generating the random number it is compared with threshold value accordingly the $\mathrm{CH}$ is chosen if the number is less than threshold value. Threshold value for normal nodes $T\left(n_{n r m}\right)$ and advance nodes $T\left(n_{a d v}\right)$ given as

$$
\begin{aligned}
\mathrm{T}\left(\mathrm{n}_{\mathrm{nrm})}=\left\{\begin{array}{cc}
\frac{\mathrm{p}_{\mathrm{nrm}}}{1-\mathrm{p}_{\mathrm{nrm}}\left[\mathrm{r} \times \bmod \left(\frac{1}{\mathrm{p}_{\text {nrm }}}\right)\right]}, \text { if } \mathrm{n} \in \mathrm{g}^{\prime} \\
0 & \text { otherwise }
\end{array}\right\}\right. \\
\mathrm{T}\left(\mathrm{n}_{\mathrm{adv}}\right)=\left\{\begin{array}{ll}
\frac{\mathrm{p}_{\text {adv }}}{1-\mathrm{p}_{\text {adv }}\left[\mathrm{r} \times \bmod \left(\frac{1}{\mathrm{p}_{\text {adv }}}\right)\right]}, & \text { if } \mathrm{n} \in \mathrm{g}^{\prime \prime} \\
0 & \text { otherwise }
\end{array}\right\}
\end{aligned}
$$

Sensor nodes with more residual energy have more chances to become $\mathrm{CH}$ than other nodes. 
Table 1. Parameters used in simulation

\begin{tabular}{|c|l|l|}
\hline Parameters & Representation & Values \\
\hline $\mathrm{n}$ & Intensity (Number) of sensor nodes & 100 \\
\hline$E_{0}$ & Initial energy of sensor nodes & $0.5 \mathrm{~J}$ \\
\hline $\mathrm{L}$ & Packet length & $4000 \mathrm{bits}$ \\
\hline $\mathrm{p}_{\mathrm{opt}}$ & Optimal Probability & 0.1 \\
\hline$E_{\text {elec }}$ & Transmitter / Receiver electronics energy & $50 \mathrm{~nJ} / \mathrm{bit}$ \\
\hline $\mathrm{E}_{\mathrm{DA}}$ & Data aggregation energy & $5 \mathrm{~nJ} / \mathrm{bit} / \mathrm{signal}$ \\
\hline$\epsilon_{f s}$ & $\begin{array}{l}\text { Energy consumed by transmit amplifier at shorter } \\
\text { distance }\end{array}$ & $10 \mathrm{pJ} / \mathrm{bit} / \mathrm{m}^{2}$ \\
\hline$\epsilon_{m p}$ & $\begin{array}{l}\text { Energy consumed by transmit amplifier at longer } \\
\text { distance }\end{array}$ & $0.0013 \mathrm{pJ} / \mathrm{bit}^{2} \mathrm{~m}^{4}$ \\
\hline$d_{0}$ & Threshold distance & $70 \mathrm{~m}$ \\
\hline $\mathrm{m} 1$ & Fraction of advance nodes for DEEC & 0.2 \\
\hline$\alpha$ & Fraction of additional energy for DEEC & 3 \\
\hline & Fraction of additional energy for IDEEC & 3 \\
\hline & & 0.3 \\
\hline
\end{tabular}

\section{SIMULATION RESULTS}

\subsection{Performance measures}

5.1.1 Network lifetime- This is based on number of half dead nodes, dead nodes and alive nodes. Half dead nodes are defined as nodes which are half dead and still half alive in comparison to dead nodes which are fully dead. Alive nodes are defined as nodes which are still alive after processing and further will participate in network operations. So there has to be increment in number of alive nodes and half dead nodes to make network alive for longer extent and decrement in number of dead nodes is required.

5.1.2 Stability (FND) - This is time till first sensor node died. The more the time interval, more is stability of network.

5.1.3 Residual Energy- This defines the amount of battery power consumed by sensor nodes per processing round. Lower the consumption and more the residual energy, better the network. 
5.1.4 Packets to $\mathbf{C H}$ - Amount of data transmitted from nodes to $\mathrm{CH}$. 5.1.5 Packets to BS- Amount of data from $\mathrm{CH}$, nodes or INs to BS.

\subsection{GUI simulation comparison}

Figure5 shows GUI (Guided user interface) showing the comparison in number of alive nodes, dead nodes in DEEC and proposed DEEC (IDEEC) routing scheme. The number of alive nodes shows increment of 38 nodes showing increase in lifetime of network. BS of DEEC was at center whereas it lies outside network field in IDEEC. The first node died at 1189 whereas it dies at 1485 in IDEEC showing increment in stability. GUI been created just to show the processing results of simulation performed in MATLAB. The network consists of 100 sensor nodes deployed randomly in $100 \mathrm{~m} * 100 \mathrm{~m}$ sensing field. The inference due to signal collisions id been avoided for performing simulation of proposed protocol IDEEC. The title of the GUI is "Advance approach of lifetime enhancement in DEEC". Two axes were considered for differentiating between DEEC and proposed IDEEC. GUI here contains push buttons, axes, static texts and variable texts.

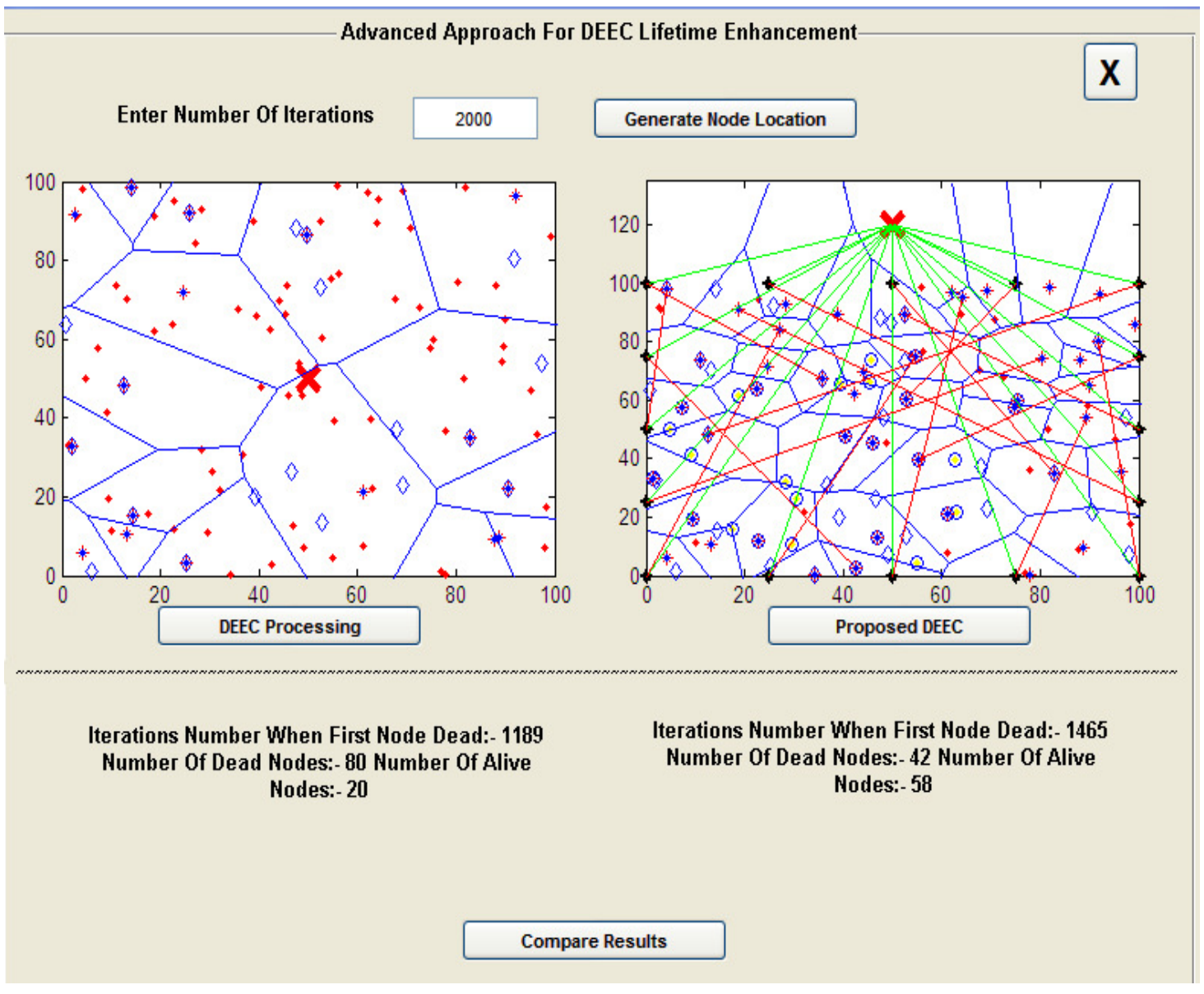

Figure 5. GUI simulation comparison 


\subsection{PERFORMANCE EVALUATION}

\subsubsection{Evaluating network lifetime:}

Figure 6 shows the number of half dead nodes in DEEC and proposed DEEC i.e. IDEEC at round 2000. The number of half dead nodes is 0 in DEEC and 28 in IDEEC. Figure 7 shows the number of normal half dead and figure 8 shows the number of advance half dead nodes in both DEECs. Initially the number of normal nodes was 80 in DEEC and 70 in IDEEC. The number of normal half dead is 0 in DEEC and 28 in IDEEC as shown in figure 7. The number of advance half dead are 0 in both DEECs showing zero improvement in number of advance half dead nodes as shown in figure 8 . Assumed number of nodes was 100 in which we have take $m=0.2$ showing number of normal nodes are 80 and number of advance nodes are 20 in DEEC and $\mathrm{m} 1=0.3$ showing number of normal nodes are 70 and number of advance nodes are 30 in IDEEC. Additional energy for DEEC and IDEEC is $300 \%$ i.e. $a=3$ been considered for both DEECs.

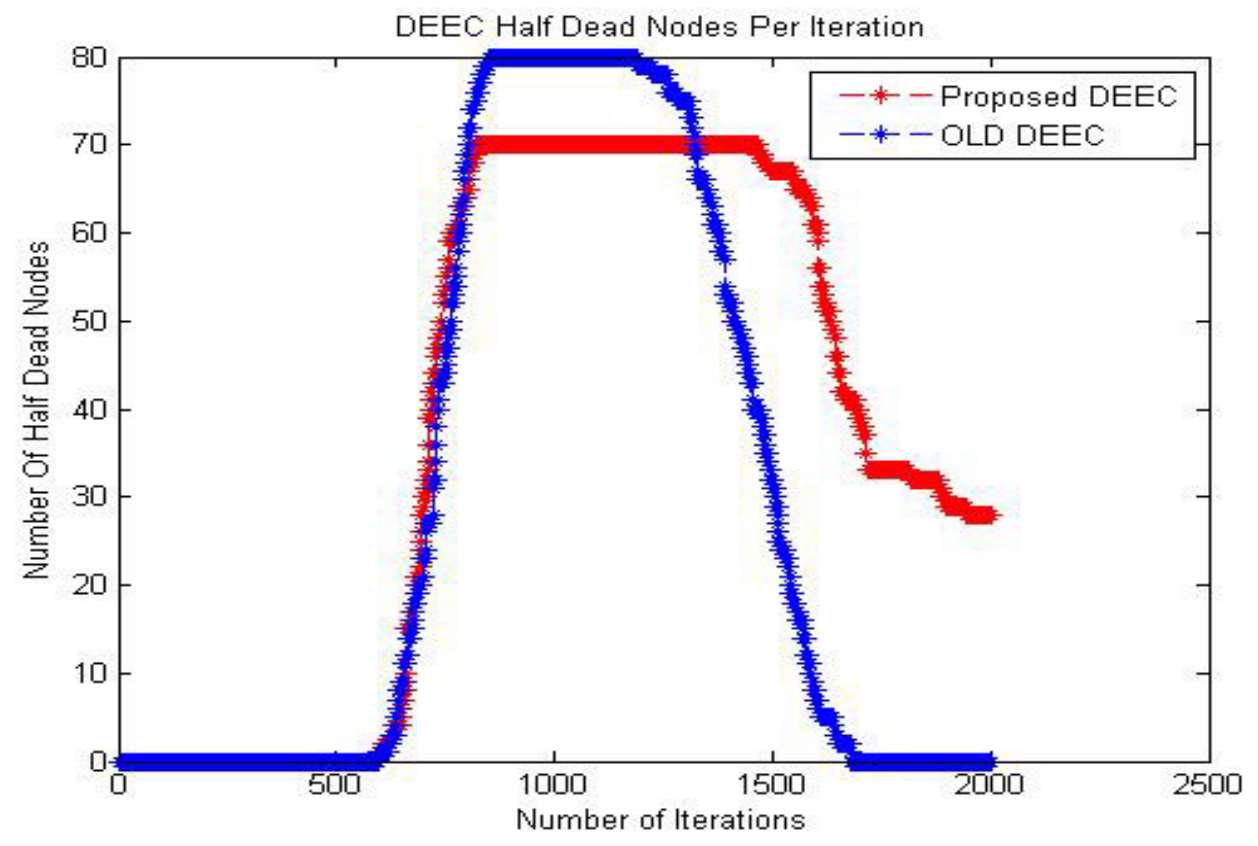

Figure 6.Number of half dead nodes 
International Journal in Foundations of Computer Science \& Technology (IJFCST) Vol.5, No.5, September 2015

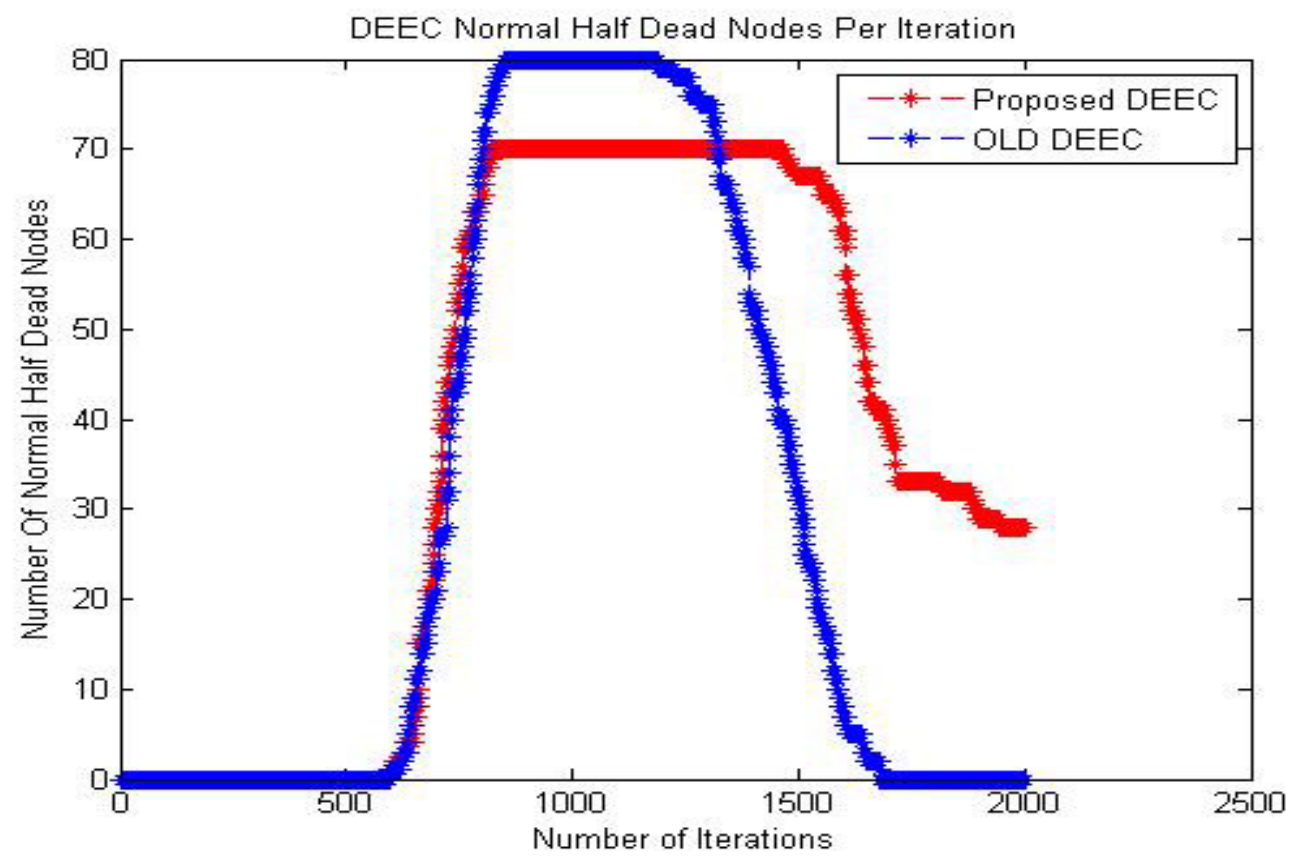

Figure 7 Number of normal half dead nodes

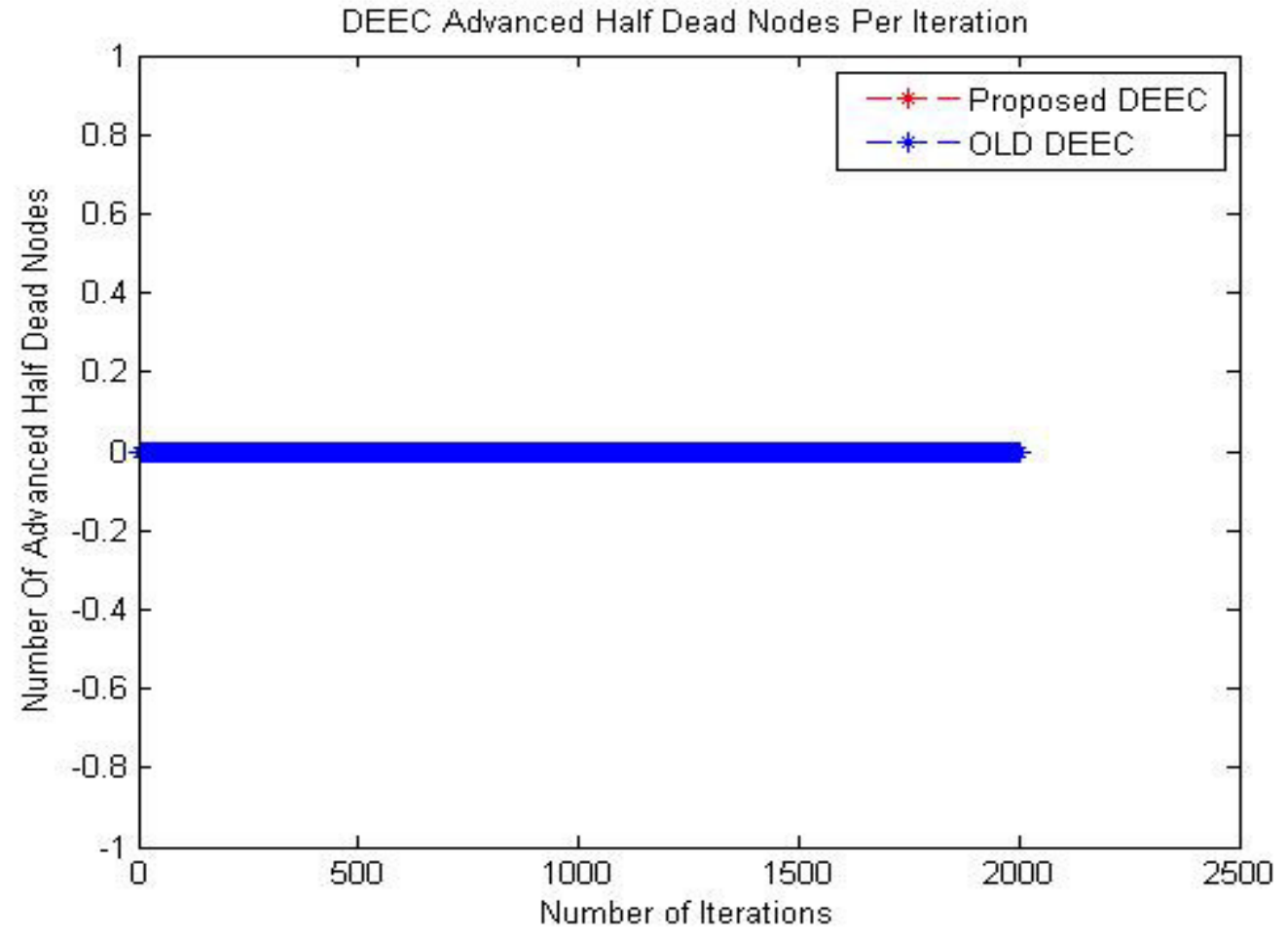

Figure 8 . Number of advance half dead nodes 
International Journal in Foundations of Computer Science \& Technology (IJFCST) Vol.5, No.5, September 2015

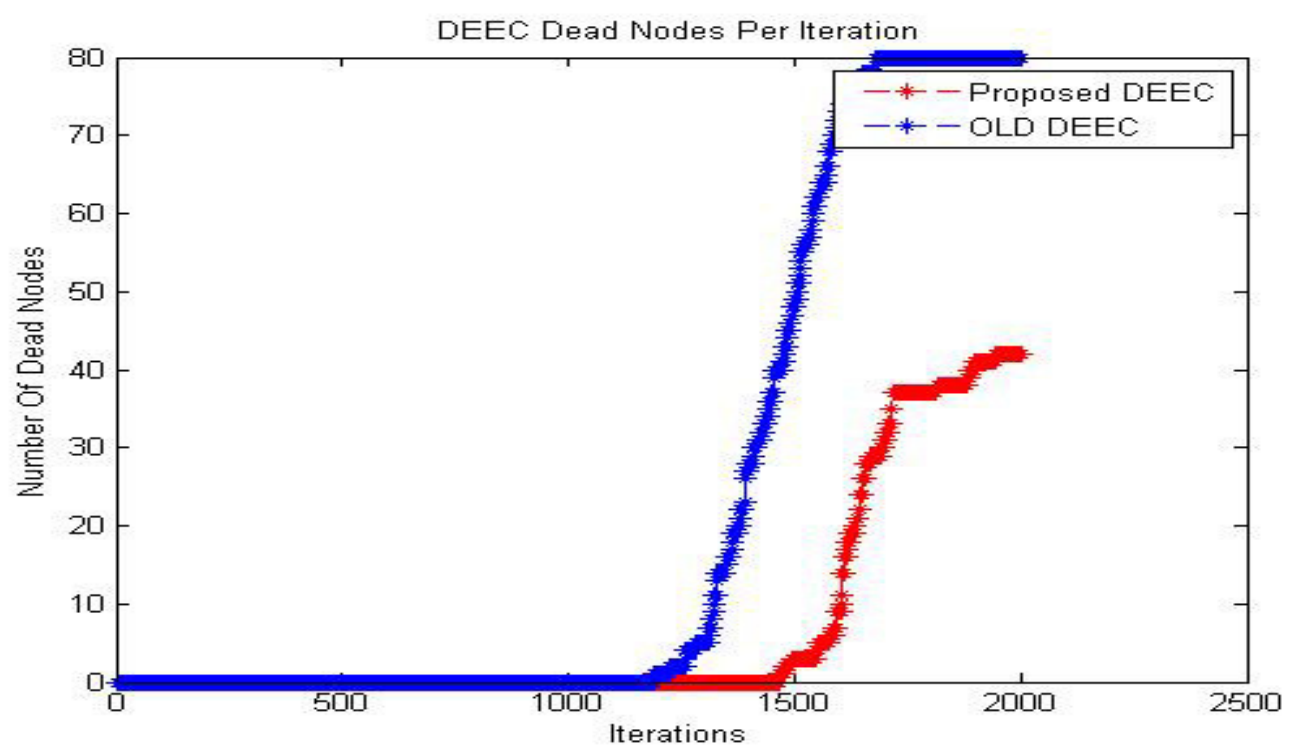

Figure 9. Number of Dead nodes

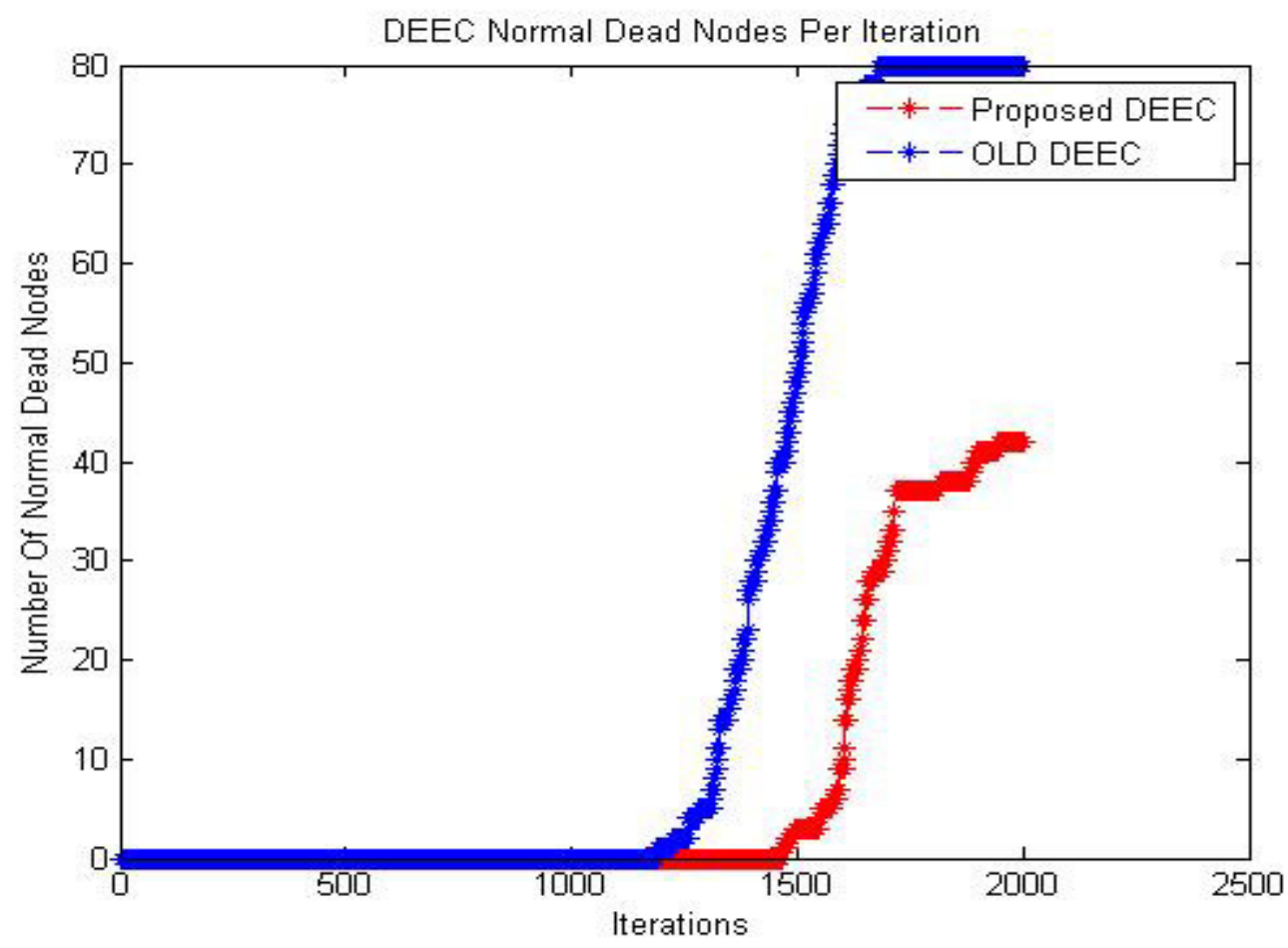

Figure 10. Number of normal dead nodes 


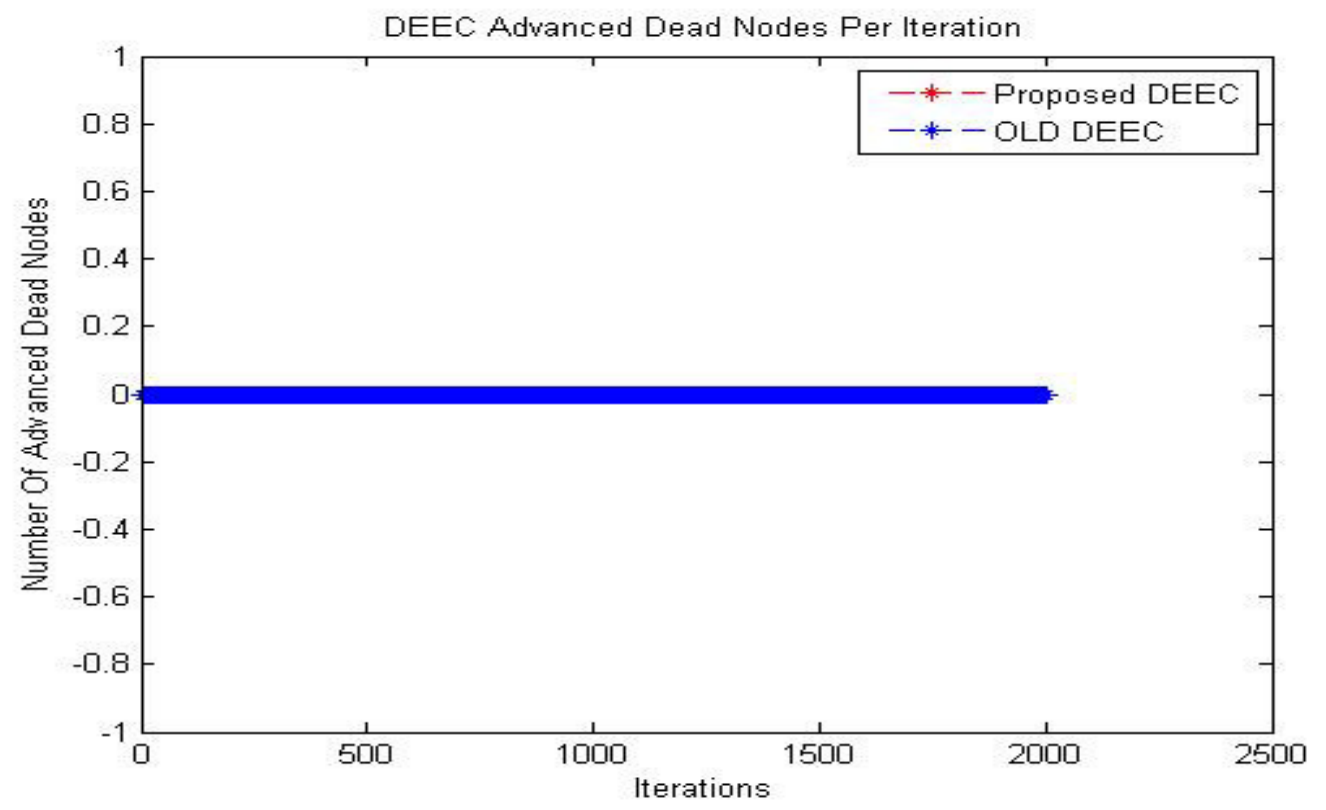

Figure 11 Number of advance dead nodes

Figure 9 shows the number of dead nodes in DEEC and proposed DEEC. The number is 80 in DEEC which is reduced to 42 in IDEEC. Initially the number of normal nodes was 80 in DEEC and 70 in IDEEC. Figure 10 shows the number of normal dead nodes which are coming to be 80 in DEEC and 42 in IDEEC. Figure 11 shows the number of advance dead nodes which are coming to be 0 in both DEECs showing no improvement. But as we increase the round number from 2000, the number of advance dead will be less in IDEEC.

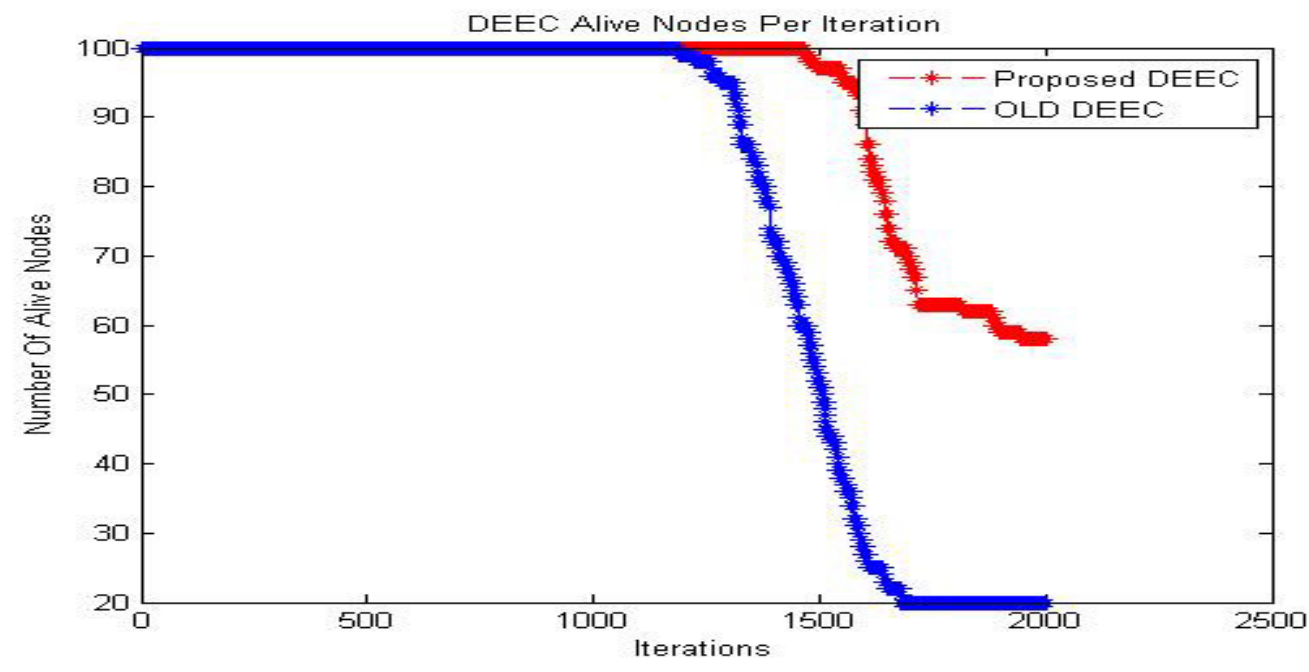

Figure 12 Number of Alive nodes 
International Journal in Foundations of Computer Science \& Technology (IJFCST) Vol.5, No.5, September 2015

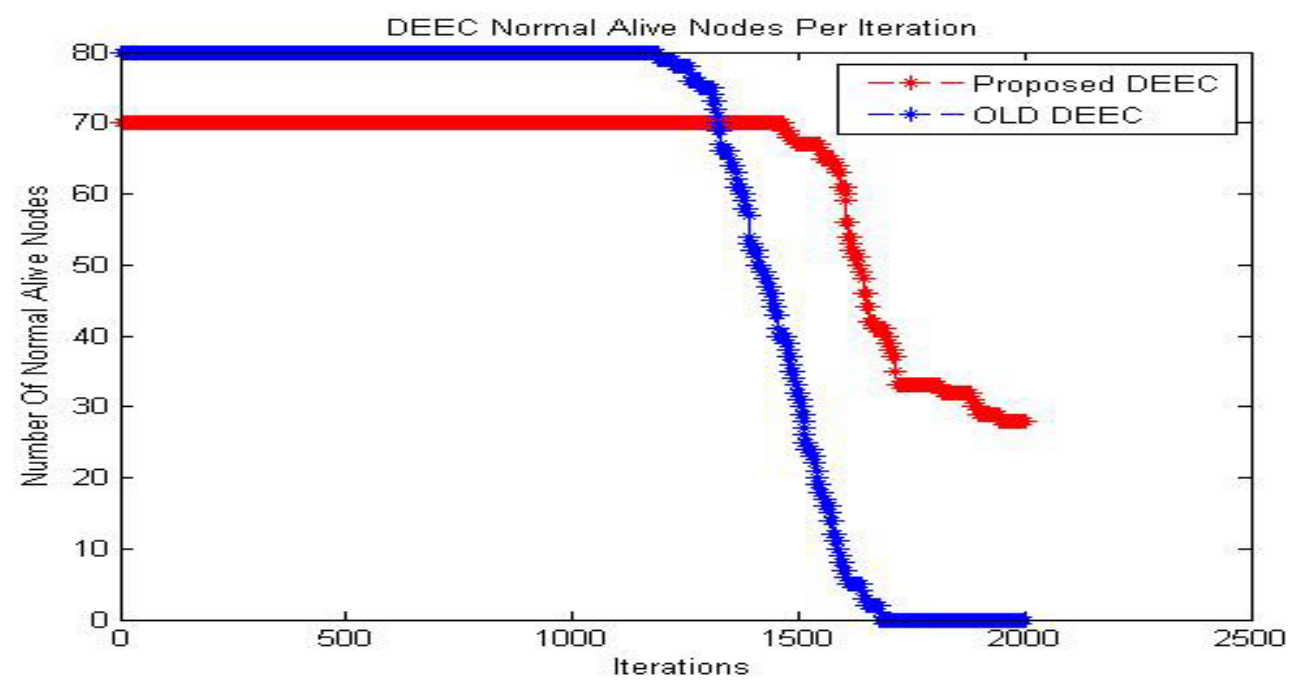

Figure 13 Number of Normal alive nodes

Figure 11 shows the number of alive nodes. The number is 20 in DEEC and 58 in IDEEC. Figure 12 shows the number of normal alive nodes which are coming to be 0 in DEEC and 28 in IDEEC. Figure 13 shows the number of advance alive nodes. The number is coming to be 20 in DEEC and 30 in IDEEC. Initially there were 20 advance nodes in DEEC and 30 in IDEEC. So there is no as such improvement in number of advance alive nodes.

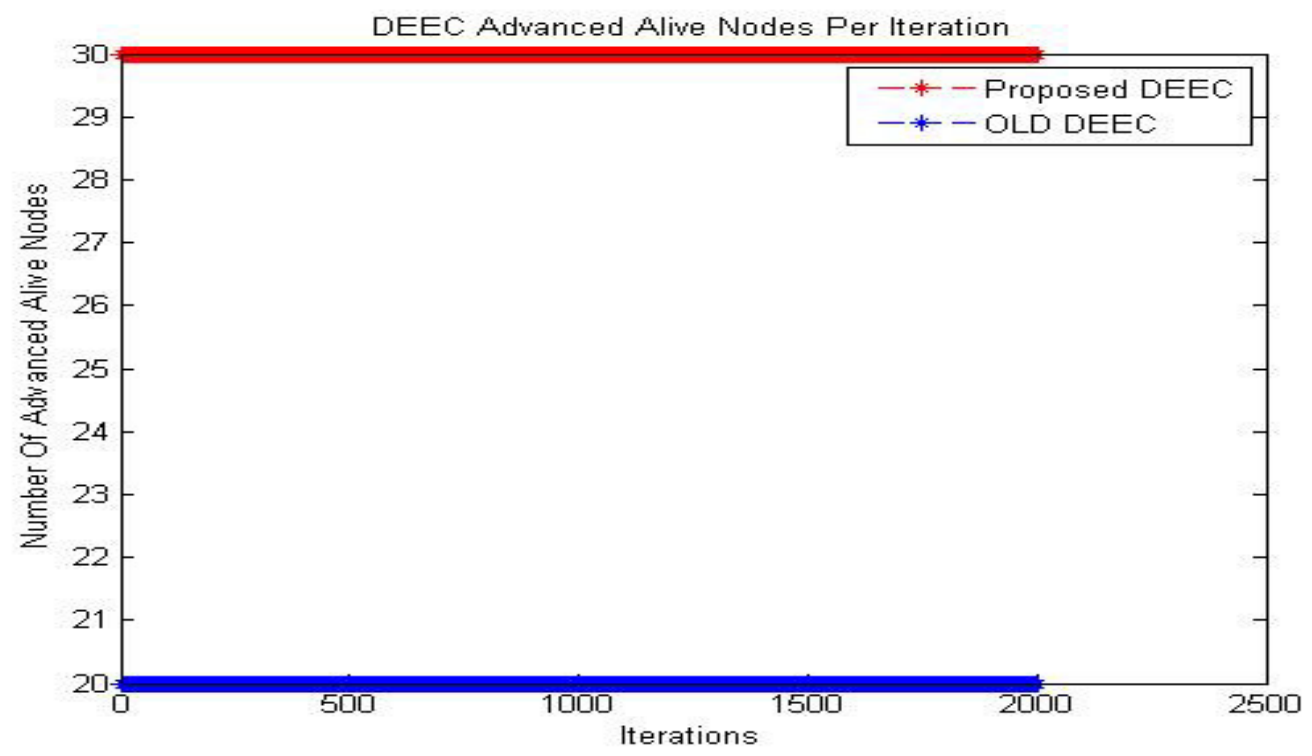

Figure 13 Number of advance alive nodes 
International Journal in Foundations of Computer Science \& Technology (IJFCST) Vol.5, No.5, September 2015

Table 2 Simulation Results of Network Lifetime

\begin{tabular}{|l|l|l|l|}
\hline $\begin{array}{l}\text { Performance measure for network lifetime } \\
\text { (In number of nodes) }\end{array}$ & DEEC & IDEEC & Improvement (\%) \\
\hline $\begin{array}{l}\text { Half Dead Nodes (Normal half dead and } \\
\text { advance half dead nodes) }\end{array}$ & 0 & 28 & 28 \\
\hline Normal Half Dead Nodes & 0 & 28 & 40 \\
\hline Advance Half Dead Nodes & 0 & 0 & 0 \\
\hline $\begin{array}{l}\text { Dead Nodes (Normal dead nodes and } \\
\text { advance dead nodes) }\end{array}$ & 80 & 42 & 38 \\
\hline $\begin{array}{l}\text { Normal Dead Nodes } \\
\text { Advance Dead Nodes }\end{array}$ & 80 & 42 & 40 \\
\hline $\begin{array}{l}\text { Alive Nodes (Normal alive nodes and } \\
\text { advance alive nodes) }\end{array}$ & 20 & 58 & 0 \\
\hline Normal Alive Nodes & 0 & 28 & 38 \\
\hline Advance Alive Nodes & 20 & 30 & 0 \\
\hline
\end{tabular}

\subsubsection{Evaluating Stability}

Figure 14 shows the time interval in form of bar graph till the first node died. The first node died much later in IDEEC than DEEC. First node died (FND) at 1189 in DEEC and 1465 in IDEEC.

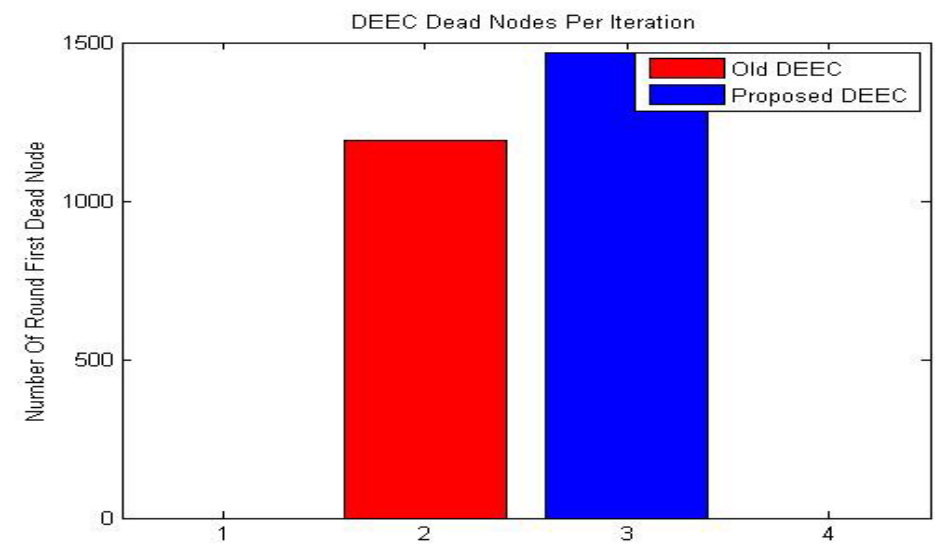

Figure 14 First Node Dead 


\subsubsection{Evaluating Residual energy}

Figure 15 shows the consumption of energy per processing round. At round 1500, there is 0.09 joules energy still left for processing in IDEEC than DEEC where there is no energy left for further processing.

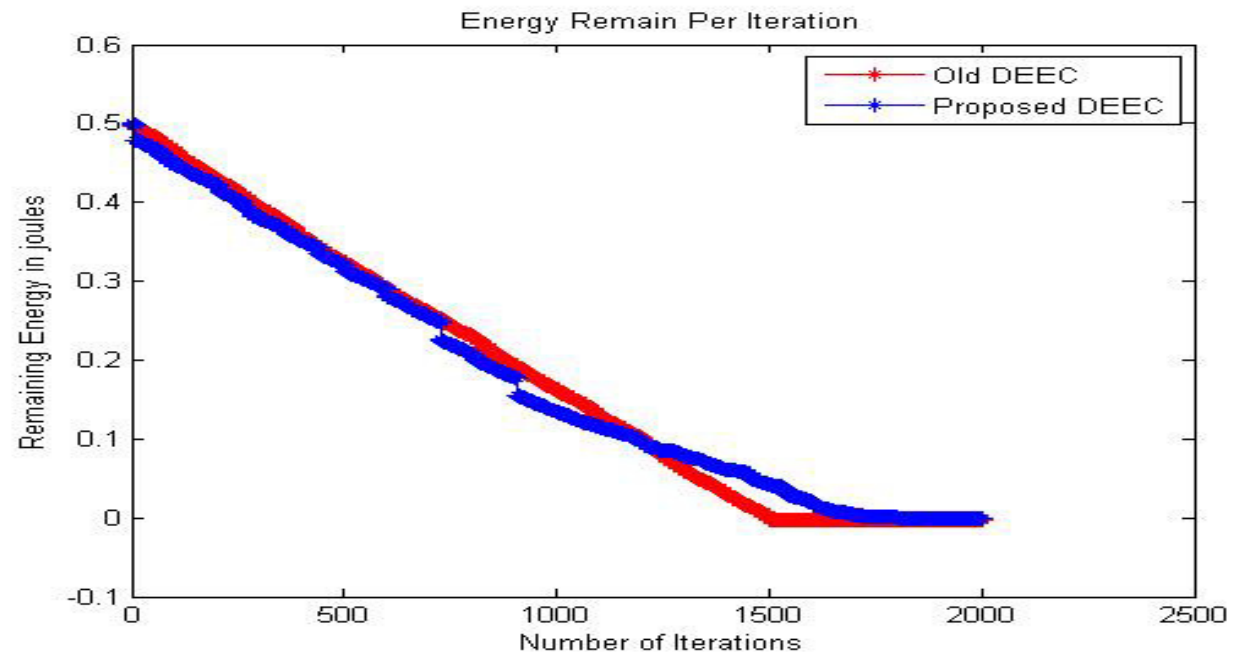

Figure 15 Residual energy

Table 3. Simulation Results of Stability and Residual Energy

\begin{tabular}{|l|l|l|l|}
\hline Performance measures & DEEC & IDEEC & Improvement (\%) \\
\hline $\begin{array}{l}\text { Stability (First node dead) } \\
\text { in round number }\end{array}$ & 1189 & 1465 & 13.8 \\
\hline $\begin{array}{l}\text { Residual Energy (joules) at } \\
1500 \text { round }\end{array}$ & 0 & 0.09 & 18 \\
\hline
\end{tabular}

\subsubsection{Packets to $\mathrm{CH}$}

Figure 16 shows the amount of packet transmission to $\mathrm{CH}$.The packet transmission is more in IDEEC. 
International Journal in Foundations of Computer Science \& Technology (IJFCST) Vol.5, No.5, September 2015

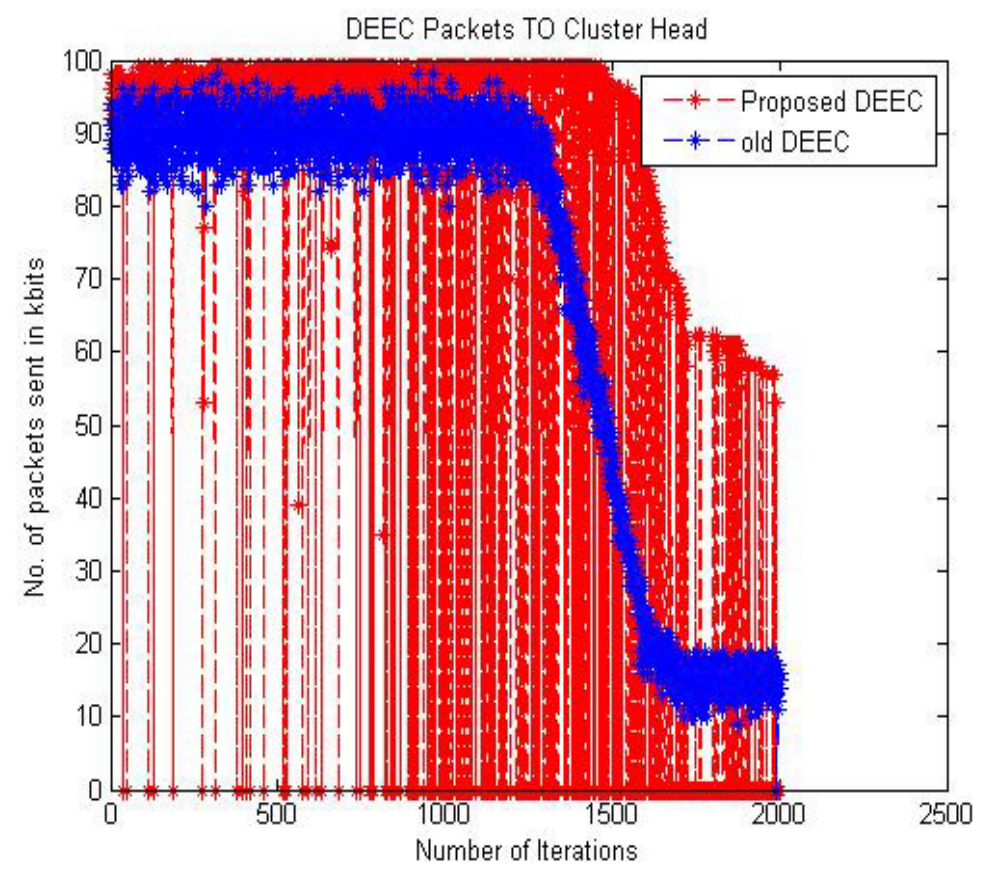

Figure 16 Packets to $\mathrm{CH}$

\subsubsection{Packets to BS}

Figure 17 shows the amount of packet transmission to BS. The amount of packet transmission is more in IDEEC than DEEC.

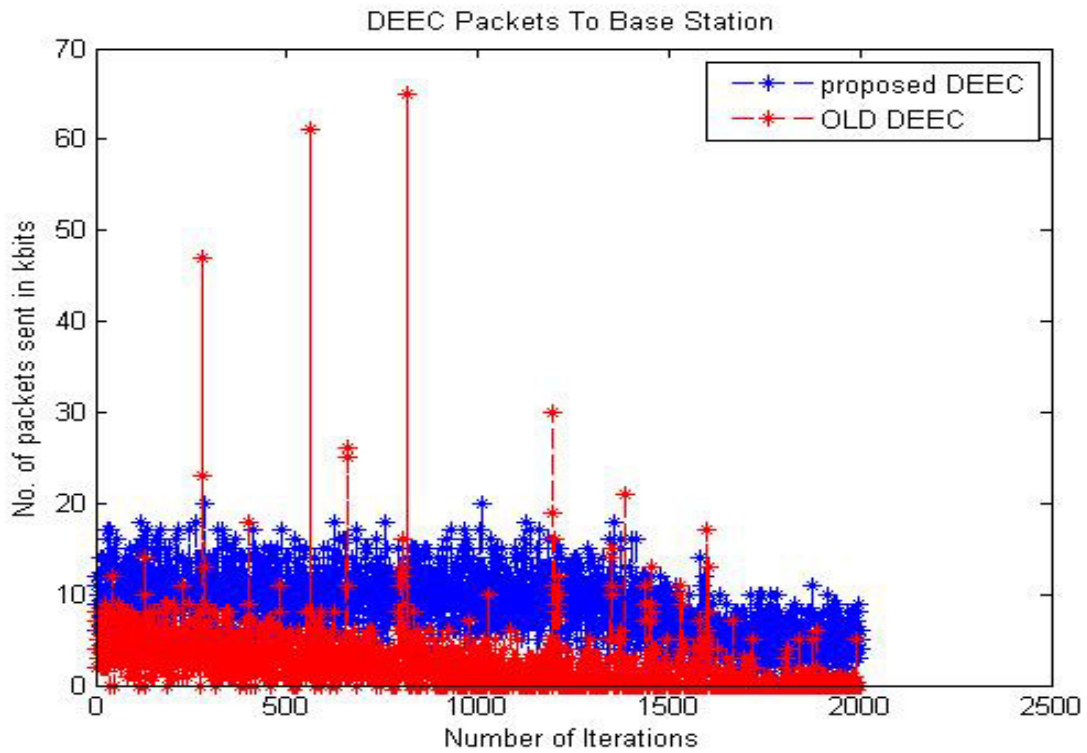

Figure 17 Packets to BS 


\section{CONCLUSIONS}

In this research a new protocol is proposed named IDEEC using intermediate nodes between $\mathrm{CHs}$ and BS. The number of half dead nodes, alive nodes, and dead nodes showed $28 \%, 38 \%, 38 \%$ improvement respectively. FND showed improvement of $13.8 \%$ and $18 \%$ improvement in residual energy. The lifetime of network increased by $34.66 \%$ on an average of three parameters i.e. half dead nodes, dead nodes and alive nodes. The BS is placed out of network field and rechargeable intermediate nodes inside the field with other nodes.

\section{REFERENCES}

[1] N. Rahmani, H. Kousha, L. Darougaran, and F. Nematy, (2010) " CAT: The New Clustering Algorithm Based on Two-Tier Network Topology for Energy Balancing in Wireless Sensor Networks," in International Conference on Computational Intelligence and Communication Networks (CICN), pp. 275-278.

[2] W. B. Heinzelman, A. P. Chandrakasan, and H. Balakrishnan, (2002) "An application-specific protocol architecture for wireless microsensor networks," Wireless Communications, IEEE Transactions on, vol. 1, pp. 660-670.

[3] L. Dehni, F. Krief, and Y. Bennani, (2006) "Power Control and Clustering in Wireless Sensor Networks," in Challenges in Ad Hoc Networking. vol. 197, pp. 31-40.

[4] W.R. Heinzelman, A. Chandrakasan and H. Balakrishnan, (2000) "Energy-efficient communication protocol for wireless microsensor networks", In Proc. International Conference on System Sciences, pp. 4-7, Maui, Hawaii.

[5] Q. Wang and I. Balasingham, (2010) "Wireless sensor networks: Application centric design", Journal of Communications, vol. 2, no. 5, pp. 134-149.

[6] S. Lindsey and C.S. Raghavendra, (2002) "PEGASIS: Power-Efficient Gathering in Sensor Information Systems", IEEE Proceedings on Aerospace, vol. 3, no.2, pp.1-6.

[7] O. Younis and S. Fahmy, (2004) "HEED: A hybrid, energy efficient, distributed clustering approach for ad hoc sensor networks", IEEE Transactions on Mobile Computing, vol. 3, no. 4, pp. 660-669.

[8] G. Smaragdakis, I. Matta and A. Bestavros, (2004) "SEP: A Stable Election Protocol for Clustered Heterogeneous Wireless Sensor Networks", In Proc. of IEEE International Workshop on SANPA, pp. $1-11$, Boston.

[9] L. Qing, Q. Zhu and M. Wang,(2006) "Design of a distributed energy-efficient clustering algorithm for heterogeneous wireless sensor networks", Journal of Computer Communications, vol. 29, no.4, pp. 2230-2237.

[10] B. Elbhiri, S. Rachid, S. El Fkihi and D. Aboutajdine, (2010) "Developed distributed energy-efficient clustering (DDEEC) for heterogeneous wireless sensor networks", In proc. IEEE 5th International Symposium on I/V Communications and Mobile Network, pp. 1-4. Rabat.

[11] S. Singh, A.K. Chauhan, S. Raghav, V. Tyagi and S. Johri, (2011) "Heterogeneous protocols for increasing lifetime of wireless sensor networks", Journal of Global Research in Computer Science, vol.2, no. 4, pp.172-176.

[12] M.M. Islam, M.A. Matin, and T.K Mondol, (2012) "Extended stable election protocol for three-level hierarchical clustered heterogeneous WSN", In Proc. of IET Conference on Wireless Sensor Systems, pp. 1-4, London, England.

[13] A.A. Khan, N. Javaid, U. Qasim, Z. Lu and Z. A. Khan, (2012) "HSEP: Heterogeneity-Aware Hierarchical Stable Election Protocol for WSNs", In Proc. IEEE International Conference on Broadband and Wireless Computing, Communication and Applications, pp. 373-378, Victoria, Canada.

[14] Q. Nadeem, M.B. Rasheed, N. Javaid, Z.A Khan, Y. Maqsood and A. Din, (2013) "M-GEAR: Gateway-Based Energy-Aware Multi-Hop Routing Protocol for WSNs", In proc. IEEE International Conference on Broadband, Wireless Computing, Communication and Applications, pp. 164-169, Compiegne, France. 
[15] Faisal, S., Javaid, N., Javaid, A., Khan, M.A., Bouk, S.H. and Khan, Z.A. (2013),“Z-SEP: ZonalStable Election Protocol for Wireless Sensor Networks", Journal of Basic and Applied Scientific Research, vol. 3, 5, pp. 132-139.

[16] C. Divya ,N. Krishnan and P. Krishnapriya,(2013) "Modified distributed energy efficient cluster for heterogeneous wireless sensor networks", In proc. IEEE Conference on Emerging trends in Computing, Communication and Nanotechnology, pp. 611-615, Tirunelveli.

[17] M.Y. Wang, J. Ding, W.P. Chen and W.Q. Guan, (2015) "SEARCH: A stochastic election approach for heterogeneous wireless sensor networks", IEEE Communication letters, vol.9, no.3, pp. 443-446.

[18] P. Jain, H. Kaur, (2014) "Gateway based stable election multi hop routing protocol for wireless sensor networks" International Journal of Mobile Network Communications \& Telematics, vol. 4, no.5, 2014. in Computing, Communication and Informatics, pp. 68-73, New Delhi, India.

\section{Authors}

SHEENAM received her Bachelor's of Technology in Electronics and Communication Engineering from SVIET, Banur affiliated to Punjab Technical University, Jalandhar, Punjab, India in 2012, and completed her degree in Masters of Technology in Electronics and Communication Engineering from Punjabi University, Patiala, Punjab, India in September 2015. She yet has published one paper on wireless sensor networks. Her research interests include routing and energy management in wireless sensor networks etc.

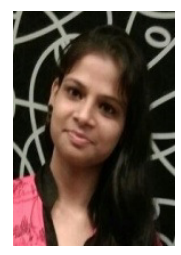

